\title{
Patient perspective and safety of remote monitoring of implantable cardioverter-defibrillators in the Polish Nationwide Multicenter Registry: the Medtronic CareLink network evaluation
}

\author{
Aleksander Maciąg' ${ }^{1}$ Przemysław Mitkowski², Michał Mazurek³, Jarosław Kaźmierczak4, \\ Krzysztof Nowak ${ }^{5}$, Marcin Grabowski ${ }^{6}$, Artur Oręziak7, Maciej Kempa ${ }^{8}$, Bogumiła Bacior ${ }^{9}$, \\ Katarzyna Gepner ${ }^{10}$, Lidia Chmielewska-Michalak², Radosław Lenarczyk³ \\ Radosław Kiedrowicz ${ }^{4}$, Artur Fuglewicz ${ }^{11}$, Andrzej Cacko ${ }^{6,12}$, Hanna Szwed ${ }^{13}$ \\ 1 2nd Department of Heart Arrhythmia, National Institute of Cardiology, Warsaw, Poland \\ 2 1st Department of Cardiology, Poznan University of Medical Science, Poznań, Poland \\ 3 1st Department of Cardiology, Congenital Heart Diseases and Electrotherapy, Silesian Center for Heart Diseases, Zabrze, Poland \\ 4 Department of Cardiology, Pomeranian Medical University, Szczecin, Poland \\ 5 Cardiology Department, Centre for Heart Diseases, Military Hospital, Wrocław, Poland \\ 1st Department of Cardiology, Medical University of Warsaw, Warsaw, Poland \\ 7 1st Department of Heart Arrhythmia, National Institute of Cardiology, Warsaw, Poland \\ 8 Department of Cardiology and Electrotherapy, Medical University of Gdańsk, Gdańsk, Poland \\ 9 Department of Coronary Artery Disease and Heart Failure, Institute of Cardiology, Jagiellonian University Medical College, John Paul II Hospital, Kraków, Poland \\ National Research Institute of Oncology in Warsaw, Warsaw, Poland \\ 1 Cardiology Department, Józef Struś City Hospital, Poznań, Poland \\ 2 Department of Medical Informatics and Telemedicine, Medical University of Warsaw, Warsaw, Poland \\ 3 Department of Cardiac Rehabilitation and Coronary Artery Disease, National Institute of Cardiology, Warsaw, Poland
}

\section{EDITORIAL}

by De Filippo et al, see p. 1086
Correspondence to: Aleksander Maciąg, MD, PhD, 2nd Department of Heart Arrhythmia, National Institute of Cardiology, ul. Alpejska 42, 04-628 Warszawa, Poland, phone: +48223434049 , email: amaciag@ikard.pl Received: May 26, 2020. Revision accepted: August 7, 2020. Published online: August 14, 2020. Kardiol Pol. 2020; 78 (11): 1115-1121 doi:10.33963/KP.15556 Copyright by the Author(s), 2020

\section{ABSTRACT}

BACKGROUND Remote monitoring of cardiovascular implantable electronic devices allows the assessment of system effectiveness, arrhythmia occurrence, and indirectly, clinical changes. Medical interventions can be performed earlier because of a faster transfer of information to the monitoring site, even in the case of asymptomatic arrhythmias or abnormalities in the operation of the system.

AIMS The aim of the study was to assess the effectiveness of remote monitoring of implantable cardioverter-defibrillators and evaluation in an outpatient setting during 12-month follow-up.

METHODS We analyzed 176 patients at 10 sites (men, 84.1\%). The mean (SD) age of the patients was 60.7 (12.5) years (range, 20-86 years), and mean (SD) follow-up period was 405 (70) days (range, $131-723$ days).

RESULTS A total of 354 outpatient and 514 remote follow-up visits were conducted. Episodes of arrhythmias and device malfunctions were detected with similar frequency in outpatient visits and in remote visits. During the study period, patient sense of safety increased. More patients preferred joined remote and outpatient visits as the optimal healthcare model. As the patient survey showed, the greatest benefit of the CareLink network was fast intervention and an increased sense of safety.

CONCLUSIONS The strategy of remote monitoring appeared to be feasible, safe, and patient friendly, demonstrating that the majority of patients do not require an additional in-person visit within 1 year from the device implantation just to confirm the proper functioning of the implantable cardioverter-defibrillators. 


\section{WHAT'S NEW?}

The majority of visits of patients with cardiovascular implantable electronic devices, both scheduled and urgent, can be successfully replaced by remote visits. An increased patient sense of security is the most important argument in favor of remote monitoring.

\section{KEY WORDS \\ implantable \\ cardioverter- \\ -defibrillator, registry, \\ remote monitoring}

number of cardiovascular implantable electronic devices (CIEDs) in use, the degree of their complexity, and elongation of life of patients with those devices have led to a dramatic increase of the system occurs during the first 6 months after the implantation. ${ }^{11}$

The primary endpoint of the study was the effectiveness of the remote monitoring of ICDs in an outpatient setting during 12-month follow-up. In addition, we evaluated the usefulness of telemedical consultation for unscheduled visits caused by distressing symptoms and high-energy interventions. in the workload of device clinics. In addition, analysis of data stored on the device and therapeutic decision-making process prolong the duration of a follow-up visit in selected patients. The guidelines on the frequency of follow-up visits in accordance with the recommendations of the European Heart Rhythm Association require the ever increasing involvement of medical personnel and suitable premises. This leads to difficulties in maintaining 6- to 12-month deadlines for follow-up visits in patients with implanted pacemakers and 3- to 6-month deadlines for follow-up visits in those with implantable cardioverter-defibrillators (ICDs). ${ }^{1}$ The majority of outpatient visits end with only a confirmation of the effectiveness of the system and do not result in any changes to the operating parameters of the device. The recommendations of international scientific societies consider the system balanced if follow-up outpatient visits are held once a year, and the rest are carried out remotely. ${ }^{1-3}$ This helps to reduce the number of outpatient visits by $30 \%$ in patients with implanted pacemakers and about $66 \%$ in patients with ICDs. As a result, more time can be devoted to the direct monitoring of patients who actually require it. Remote monitoring allows the assessment of system effectiveness (analysis of the basic electrical parameters of the system), arrhythmia occurrence, and indirectly, clinical changes. Medical interventions can occur earlier because of the more rapid transfer of information to the monitoring site, even in the case of asymptomatic arrhythmias or abnormalities in system operation. Even early studies analyzing the effectiveness of remote device monitoring care show that there was no deterioration in patient prognosis. Moreover, there was a reduction in inadequate discharges, prolonged battery life, and earlier diagnosis of abnormalities in system operation. Also, asymptomatic atrial fibrillation was detected more often and suitable anticoagulation therapy was implemented effectively. ${ }^{4-8}$ More recent registry and randomized studies indicate the benefits associated with a better prognosis (survival) in patients monitored remotely. ${ }^{9-10}$ Virtually, the only limit to remote device monitoring is the inability to make changes to the device program. In most cases, the need to change the parameters
METHODS A multicenter nonrandomized prospective registry was conducted at 10 sites in Poland. The study protocol was approved by the bioethics committee of the Institute of Cardiology in Warsaw.

The study included patients who, in a period from 30 days to 1 year before joining the registry, received an ICD or a cardiac resynchronization therapy device (CRT-D) enabling communication with the CareLink network (Medtronic, Minneapolis, Minnesota, United States). Every patient gave their informed consent. The exclusion criteria were as follows: younger than 18 years of age, noncompliance, or lack of consent to participate in the program.

The study protocol for each patient included clinic (at the beginning and end of the study), and remote device monitoring through the CareLink network in the following periods: 3 to 4,6 to 8 , and 9 to 12 months of the follow-up period. Outpatient and remote visits were performed by a physician participating in the study. $\mathrm{Pa}$ tients were also asked to contact the site in case of any distressing symptoms or the delivery of high-energy therapy. All unscheduled followic were additionally recorded. Additional monitoring requested during any type of visit was classified as a continuation of a previous visit and not a new episode. During the visits, data were collected on arrhythmia, detected abnormalities, decisions made, methods of modifying the therapy, and patient subjective evaluation of the CareLink network. After the first and the last transmission in the study, feasibility and acceptance of the remote monitoring model of care were evaluated in a survey completed by the patients.

Statistical analysis Statistical analysis was performed with the SAS 8.2 (SAS, Institute Inc, Cary, North Carolina, Unites States). Continuous data with normal distribution were presented as means (SD) and data with nonnormal distribution, as medians and interquartile ranges. The frequency and percentage of distinguished units of nominal features were also used. The nominal variables were assessed with contingency tables, and the distribution of features was analyzed first by the $\mathrm{X}^{2}$ test. In cases where the expected both outpatient visits at a device monitoring -up visits performed remotely and at the clin- 


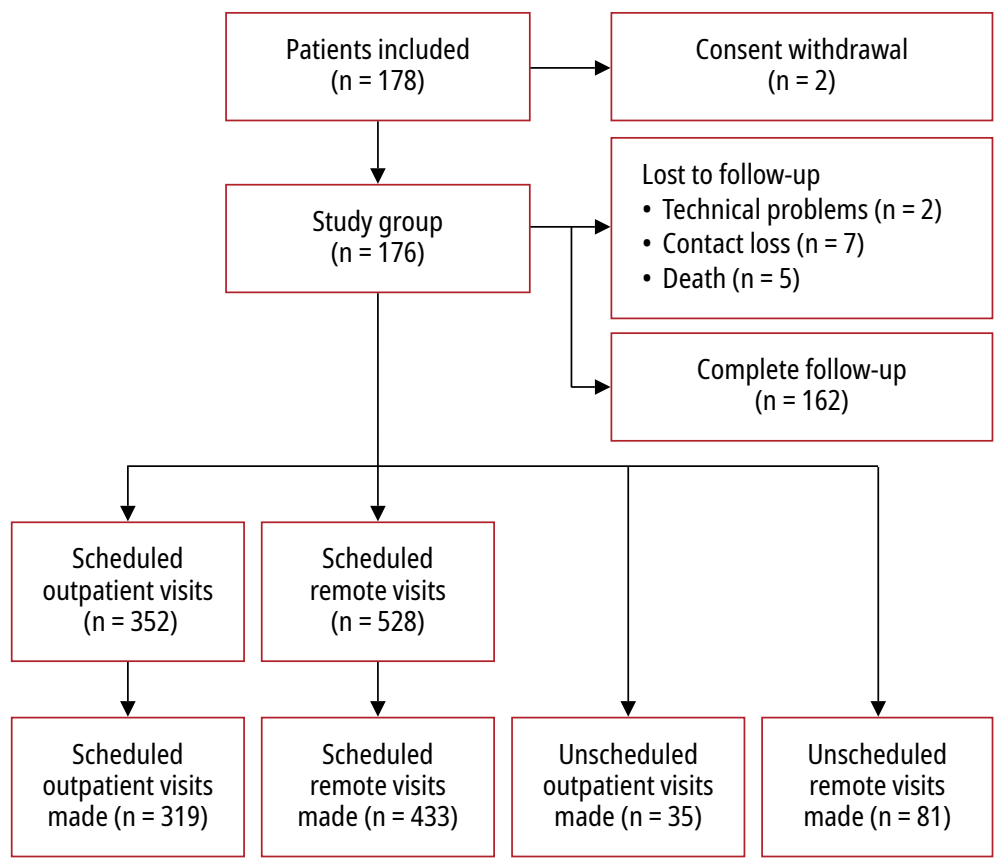

FIGURE 1 Flow chart of patients and visits
Visits For each patient, telemetric visits and outpatient visits were planned in a 3 to 2 ratio. In total, $91 \%$ of the 352 scheduled outpatient visits and $82 \%$ of the 528 scheduled remote visits were carried out. This translates into 752 visits preformed scheduled in the protocol, of which 319 (42.4\%) were carried out at the outpatient clinic and 433 (57.6\%), remotely. The remaining 116 visits (13.4\%) were unscheduled visits, including 35 (30.2\%) outpatient and 81 (69.8\%) remote visits. (FIGURE1). Patients who participated in the remote unscheduled visits were older than those in outpatient visits (mean [SD] age, 61.5 [11.1] years and 54.7 [15.7] years, respectively; $P=0.02$ ).

During the scheduled visits, episodes of arrhythmias, both ventricular and supraventricular, were detected with similar frequency in the outpatient visits and remote visits (101 [31.7\%] and 159 [36.7\%], respectively; $P=0.15$ ). Ventricular arrhythmias meeting the detection criteria were found during 35 (11\%) outpatient visits and 54 (12.5\%) remote visits. During 18 (2.4\%) visits, both ventricular and supraventricular arrhythmias were recorded. The majority of arrhythmic episodes (93.5\%) were correctly treated by the device ( $94.7 \%$ for outpatient visits and $92.7 \%$ for remote visits, respectively). In the case of outpatient visits, incorrect diagnosis was related to ventricular arrhythmias in only 2 patients $(0.6 \%)$, including 1 case that required lowering the ventricular tachycardia detection threshold. Incorrect diagnosis occurred in 4 cases (16\%) and did not require changes to the program but only a recommendation to change the drug therapy. According to physicians who monitored data stored in the device memory, these data allowed for the correct assessment of arrhythmia in all patients during both outpatient and remote visits.

During the unscheduled outpatient and remote follow-up visits, arrhythmias, both ventricular and supraventricular, were detected more often than during scheduled visits. Arrhythmias occurred during 15 (42.9\%) out of 35 outpatient visits and 40 (49.4\%) out of 81 remote visits, respectively. Ventricular arrhythmias meeting the detection criteria were identified during 8 outpatient visits (11\%) and 54 remote visits (12.5\%). There were no differences in the incidence of arrhythmia between the outpatient and remote visits. Both ventricular and supraventricular arrhythmias were recorded during 3 visits (2.6\%). Most of arrhythmic episodes (77\%) were correctly treated by the device, although this percentage was lower than in scheduled visits. The percentages were $66.7 \%$ for outpatient visits and $80.4 \%$ for remote visits (in comparison with the scheduled visits, the differences were $P<0.01$ for outpatient visits and $P=0.02$ for remote visits). In the case of outpatient visits, incorrect diagnosis and procedure were related to ventricular arrhythmia in 


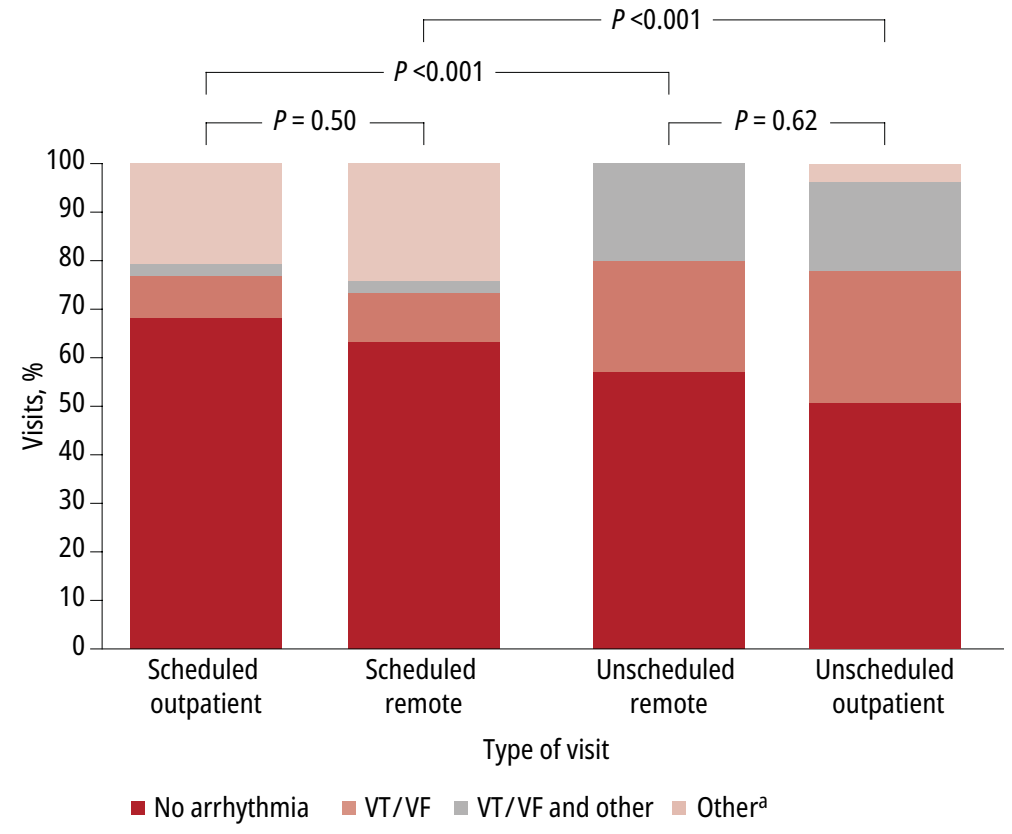

FIGURE 2 Frequency of arrhythmia detection during implantable cardioverter-defibrillator (ICD) monitoring, both outpatient and remote, scheduled $(n=752)$ and unscheduled $(n=116)$ visits.

a Other included nonsustained ventricular tachycardia, supraventricular tachycardia, atrial tachycardia, and atrial fibrillation

Abbreviations: VT/VF, ventricular tachycardia / ventricular fibrillation

one case $(6.7 \%)$ and was the reason for changing the ICD program, and in the case of remote visits, this occurred in 4 cases $(16 \%)$ and did not require changes in the program but only a recommendation to change the drug therapy in 2 cases (FIGURE2).

As for the scheduled visits, device malfunctions were detected both during outpatient and remote visits only in few patients, that is,
$3(0.9 \%)$ and $4(0.9 \%)$, respectively. In the case of remote visits these included: electromagnetic interference on the atrial electrode recorded by the device as arrhythmia (1 patient), T-wave oversensing (1 patient), and resistance alarm in 2 patients. In the case of the outpatient visits, there was a threshold increase in 1 patient and damage to an electrode in 2 patients. As for the unscheduled visits, there was only a single case of T-wave oversensing (0.9\%) during a remote visit, which caused an additional outpatient visit.

During the scheduled visits, a necessity for a medication change, additional visit, or hospitalization resulting from the above observations of the device operation or arrhythmia was recorded with a similar frequency during outpatient and remote visits. Malfunctions of the device requiring additional outpatient checks were detected only during 6 outpatient visits (1.4\%). Additional checks of the device were necessary in 1 outpatient visit (0.3\%). Device reprogramming or referring for further diagnostics occurred more frequently. During unscheduled visits, the percentage of ordered interventions was comparable in remote and outpatient visits, except for device reprogramming, which took place more often during outpatient visits. The comparison of scheduled and unscheduled visits shows that a higher percentage of patients on unscheduled visits required intervention with respect to programming, medication change, and additional visits. A detailed comparison is presented in TABLE 1.

The most frequently reported reasons for remote additional outpatient visits were alarming symptoms, device alert, or patient anxiety,

TABLE 1 Interventions resulting from data collected during visits (more than 1 intervention in 6 patients of scheduled and 7 unscheduled visits)

\begin{tabular}{|c|c|c|c|c|c|c|c|c|}
\hline \multirow[t]{2}{*}{ Intervention } & \multicolumn{3}{|c|}{ Scheduled visits } & \multicolumn{3}{|c|}{ Unscheduled visits } & \multicolumn{2}{|c|}{$\begin{array}{l}P \text { value (scheduled vs } \\
\text { unscheduled) }\end{array}$} \\
\hline & $\begin{array}{l}\text { Outpatient } \\
(n=319)\end{array}$ & $\begin{array}{l}\text { Remote } \\
(n=433)\end{array}$ & $P$ value & $\begin{array}{l}\text { Outpatient } \\
(n=35)\end{array}$ & $\begin{array}{l}\text { Remote } \\
(n=81)\end{array}$ & $P$ value & Outpatient & Remote \\
\hline No intervention & $275(86.2)$ & $389(89.8)$ & 0.13 & $24(68.6)$ & $56(69.1)$ & 0.95 & 0.006 & $<0.001$ \\
\hline Reprogramming & $25(7.8)$ & $1(0.2)$ & $<0.001$ & $7(20)$ & 0 & $<0.001$ & 0.03 & $>0.99$ \\
\hline $\begin{array}{l}\text { Ordering further } \\
\text { diagnostics }\end{array}$ & $4(1.2)$ & 0 & 0.03 & $1(2.9)$ & $2(2.5)$ & $>0.99$ & 0.4 & 0.02 \\
\hline $\begin{array}{l}\text { Medication } \\
\text { change }\end{array}$ & $12(3.8)$ & $27(6.2)$ & 0.13 & $3(8.6)$ & $10(12.3)$ & 0.75 & 0.18 & 0.05 \\
\hline $\begin{array}{l}\text { Additional } \\
\text { outpatient control }\end{array}$ & $1(0.3)$ & $6(1.4)$ & 0.25 & $2(5.7)$ & $3(3.7)$ & 0.64 & 0.03 & 0.16 \\
\hline $\begin{array}{l}\text { Additional } \\
\text { consultation }\end{array}$ & $4(1.25)$ & $4(0.9)$ & 0.73 & 0 & $5(6.2)$ & 0.32 & $>0.99$ & 0.006 \\
\hline $\begin{array}{l}\text { Admission to } \\
\text { hospital }\end{array}$ & $5(1.6)$ & $1(0.2)$ & 0.09 & $1(2.9)$ & $7(8.6)$ & 0.43 & 0.47 & $<0.001$ \\
\hline Others & $1(0.3)$ & $8(1.9)$ & 0.09 & $1(2.9)$ & $1(1.2)$ & 0.51 & 0.19 & $>0.99$ \\
\hline
\end{tabular}


TABLE 2 Causes of additional visits (multiple choice answers: in 14 patients there was more than 1 [2 or 3] cause); groups are not separable

\begin{tabular}{|c|c|c|c|c|}
\hline Cause of additional visit & Total & Outpatient clinic/hospital & Remote & $P$ value \\
\hline Symptoms reported by patients & $31(26.7)$ & $5(14.3)$ & $26(32.1)$ & 0.046 \\
\hline Appropriate discharge & $17(14.7)$ & $4(11.4)$ & $13(16.1)$ & 0.52 \\
\hline Inappropriate discharge & $7(6)$ & $2(5.7)$ & $5(6.2)$ & $>0.99$ \\
\hline Device alert & $20(17.2)$ & $1(2.9)$ & $19(23.5)$ & 0.007 \\
\hline Necessity of reprogramming & $2(1.7)$ & $2(5.7)$ & 0 & 0.09 \\
\hline Patient anxiety & $19(16.4)$ & $1(2.9)$ & $18(22.2)$ & 0.01 \\
\hline Deteriorated well-being & $1(0.9)$ & 0 & $1(1.2)$ & $>0.99$ \\
\hline Assessment of heart rate & $2(1.7)$ & 0 & $2(2.5)$ & $>0.99$ \\
\hline During outpatient visit due to another reason & $34(29.3)$ & $25(71.4)$ & $9(11.1)$ & $<0.001$ \\
\hline Arrhythmias without ICD intervention & $3(2.6)$ & 0 & $3(3.7)$ & 0.55 \\
\hline Stimulation disturbances & $1(0.9)$ & $1(2.9)$ & 0 & 0.3 \\
\hline
\end{tabular}

Data are presented as number (percentage).

Abbreviations: ICD, implantable cardioverter-defibrillator

TABLE 3 Patient subjective sense of safety

\begin{tabular}{lll} 
Sense of safety & Week 1 & Month 12 \\
\hline Much safer & $40(30.5)$ & $66(44.6)$ \\
\hline Safe & $75(57.3)$ & $75(50.7)$ \\
\hline No influence & $15(11.4)$ & $7(4.7)$ \\
\hline Unsafe & $1(0.8)$ & 0 \\
\hline
\end{tabular}

Data are presented as number (percentage).

TABLE 4 Patient opinion on the benefits of using a telemedical system to control ICDs (multiple choice answers, groups are not separable)

\begin{tabular}{llll} 
Opinion & Week $1(n=132)$ & Month $12(n=147)$ & $P$ value \\
Fast intervention in case of problems & $98(74.2)$ & $99(67.4)$ & 0.21 \\
\hline Security/safety & $59(44.7)$ & $60(40.8)$ & 0.51 \\
\hline Time saving & $45(34.1)$ & $43(29.2)$ & 0.39 \\
\hline Cost saving & $28(21.2)$ & $25(17)$ & 0.37 \\
\hline Flexibility & $21(15.9)$ & $21(14.3)$ & 0.71 \\
\hline Other & $4(3)$ & $24(16.3)$ & 0.001 \\
\hline
\end{tabular}

Data are presented as number (percentage).

with a total of $77.7 \%$ of all reports. A detailed description of the reasons for the visits, with division into outpatient or hospital and remote visits, is presented in TABLE 2.

People monitoring the devices claimed that in the case of additional remote visits, as many as 31 cases (38.3\%) would require additional outpatient visits, unless a remote visit could be performed. On the other hand, in the case of additional outpatient visits, as many as 25 cases $(75.8 \%)$ could have been carried out remotely.
Subjective assessment of the CareLink network The majority of patients, both during the first and last transmission, found the handling of the monitor easy or very easy. After the first transmission, there were 125 such answers (94.7\%) out of 132, and after the last transmission, 139 (93.3\%) out of 149. This assessment did not change in the course of the program. Only 16 patients (12.1\%) during the first and 25 patients $(16.8 \%)$ during the last transmission required the assistance of other people. It 
also appears that the CareLink network provided a sense of safety, observed in the follow-up period. The majority of patients stated that their sense of safety increased (Fisher exact test, $P=0.02$ for trend) (tABle 3 ).

Experience with the CareLink network resulted in more patients preferring joined remote and outpatient visits as the optimal healthcare model. After the first transmission, there were 117 such answers out of 132, and after the last transmission, 107 out of $149,88.6 \%$ and $71.8 \%$, respectively. The group of patients with no preference increased.

As the patient survey showed, the greatest benefit of the CareLink network was fast intervention in case of problems and an increased sense of safety. This did not change during the whole study period. A lower number of patients also reported additional benefits such as saving time and costs related to the visit (TABLE4).

DISCUSSION The main finding of this study is that remote control of ICDs and CRT-Ds with the Medtronic CareLink network is feasible and safe, and thus, allows to carry out routine outpatient visits once a year in patients with CIEDs. No differences between the conventional and remote visits were observed with regard to proper detection of arrhythmic events as well as to the diagnosis of any device malfunctions throughout the whole follow-up period. The majority of scheduled visits were carried out successfully both in outpatient visits and remotely (91\% and $82 \%$, respectively). According to the opinion of physicians who performed the conventional 1 year follow-up visit at outpatient clinics, as many as $75.8 \%$ of telemetric visits would have been sufficient and satisfactory as the only way of monitoring CIEDs. These findings were in line with the current Heart Rhythm Society Expert Consensus Statement on remote interrogation and monitoring for CIED's. ${ }^{12}$

Secondly, as many as $13.4 \%$ of all study visits were unscheduled and, even more significantly, $69.8 \%$ of them were telemetric. Of note, the majority of unscheduled visits were patient-initiated as a result of anticipated symptoms, anxiety, or device alerts. Importantly, about a third of these unscheduled transmissions required medication change, additional consultation, outpatient visit, or even hospital admission $(12.3 \%, 6.2 \%, 3.7 \%$, and $8.6 \%$, respectively). This reflects one of the greatest advantages of the Medtronic CareLink network, which allows every patient to actively participate in remote monitoring of the transmissions at every opportunity. Of note, patients who initiated an unscheduled transmission were older than those who waited until the scheduled visits (mean [SD] age, 61.5 [11.1] years and 54.7 [15.7] years, respectively). Taking into consideration the fact that for over $96 \%$ of patients the use of the monitor was easy or very easy, it should not be a surprise that over $95 \%$ of the subjects felt safe or even more safe with the CareLink network. These findings are of particular importance as many patients with heart failure, in particular those with implantable devices, have depression and anxiety, and thus, their active participation in the treatment may exert a positive effect on the understanding of the disease as well as long-term outcomes. ${ }^{13-16}$

Third, scheduled remote transmissions, which were performed in line with the study protocol every 3 months, resulted in no intervention in $89.9 \%$ of cases. The most frequent intervention was medication change or dose up-titration (6.2\%), while additional in-person consultations or acute hospital admissions were very infrequent $(0.9 \%$ and $0.2 \%$, respectively). Of note, the need for device reprogramming was necessary only in $0.2 \%$ of cases. These findings confirm that the majority of patients do not require routine visits to the outpatient clinic every 3 to 6 months just to confirm the proper functioning of the implanted devices.

Several recent studies confirmed that remote monitoring of implantable devices is not only safe and feasible but also allows early diagnosis of any device malfunctions, reduces the number of in-office visits a well as both inappropriate and appropriate shocks, and eventually contributes to lower costs of treatment. ${ }^{4-7,17-20}$ Moreover, it has been recently proven that remote monitoring reduces mortality in patients with heart failure. These fundamental findings were initially observed in large nonrandomized observational registries, like ALTITUDE or MERLIN, 6,21 and finally confirmed in a prospective, randomized clinical trial, Implant-Based Multiparameter Telemonitoring of Patients with Heart Failure (IN-TIME). ${ }^{10}$ It was shown for the first time that daily, implant-based remote monitoring improves survival in comparison with conventional outpatient follow-up visits in patients with CIEDs. ${ }^{10}$ What is more, these observations seem to be true for remote follow-up visits for different kinds and modalities of CIEDs, including pacemakers, and that the real advantage of remote patient monitoring depends directly on both the adherence to therapy and time spent on being constantly monitored. ${ }^{21}$ A recent study also suggested that remote monitoring of patients with heart failure with implanted ICDs or CRT-Ds reduced the hospitalization rate in the remote monitoring care arm. ${ }^{22}$ These observations, which reinforce the role of remote monitoring, were strongly supported by the recently published opinion of Polish experts of telemedicine. ${ }^{23}$

Limitations The main limitation of this study is lack of comparison between remote and conventional follow-up. Moreover, analyzing remote 
monitoring provided by only 1 company might affected comparison between remote monitoring systems from different manufacturers.

Conclusions The strategy of remote monitoring appeared to be feasible, safe, and patient friendly, demonstrating that the majority of patients do not require an additional in-person visit within 1 year just to confirm the proper functioning of the device. The trial revealed moreover that more than two-thirds of the unscheduled visits were telemetric and in a third of cases additional medical intervention was required. As this trial did not compare patients monitored remotely with those who were followed in a conventional way, since all the study patients were equipped with and used the Medtronic CareLink network transmitter, we cannot make a conclusion with respect to the superiority of any of the methods presented in this study. However, bearing in mind that no major issues concerning the remote monitoring appeared throughout the whole study period, the telemetric control of CIEDs could become the routine type of follow-up care in Poland.

\section{ARTICLE INFORMATION}

ACKNOWLEDGMENTS This work was supported by Medtronic Poland as a Medtronic CareLink Network Evaluation Registry (ClinicalTrials.gov identifier, NCT01023022).

CONFLICT OF INTEREST All authors were included in the Medtronic CareLink Network Evaluation Registry (ClinicalTrials.gov identifier, NCT01023022). AM received lecture fees and travel grants from St. Jude Medical, Medtronic, and Biotronik. PM received lecture fees and travel grants from Medtronic. MM received lecture fees and travel grants from Medtronic. MG received lecture fees and travel grants from Medtronic. MK received travel grants from Medtronic. RL received lecture fees from Medtronic, Biotronik, and St. Jude Medical.

OPEN ACCESS This is an Open Access article distributed under the terms of the Creative Commons Attribution-NonCommercial-NoDerivatives 4.0 International License (CC BY-NC-ND 4.0), allowing third parties to download articles and share them with others, provided the original work is properly cited, not changed in any way, distributed under the same license, and used for noncommercial purposes only. For commercial use, please contact the journal office at kardiologiapolska@ptkardio.pl.

HOW TO CITE Maciąg A, Mitkowski P, Mazurek M, et al. Patient perspective and safety of remote monitoring of implantable cardioverter-defibrillators in the Polish Nationwide Multicenter Registry: the Medtronic CareLink network evaluation. Kardiol Pol. 2020; 78: 1115-1121. doi:10.33963/KP.15556

\section{REFERENCES}

1 Wilkoff BL, Auricchio A, Brugada J, et al. HRS/EHRA Expert Consensus on the monitoring of cardiovascular implantable electronic devices (CIEDs): description of techniques, indications, personnel, frequency and ethical considerations. Europace. 2008: 10: 707-725.

2 Dubner S, Auricchio A, Steinberg JS, et al. ISHNE/EHRA expert consensus on remote monitoring of cardiovascular implantable electronic devices (CIEDs). Europace. 2012; 14: 278-293.

3 Piotrowicz R, Grabowski M, Balsam P, et al. "Baltic Declaration" - telemedicine and $m$ Health as support for clinical processes in cardiology. The opinion of the Committee of Informatics and Telemedicine of the Polish Society of Cardiology and Telemedicine Clinical Sciences Committee of the PAS. Kardiol Pol. 2015; 73: $575-584$.

4 Guedon-Moreau L, Lacroix D, Sadoul N, et al. A randomized study of remote follow-up of implantable cardioverter-defibrillators: safety and efficacy report of the ECOST trial. Eur Hear J. 2013; 34: 605-614.

5 Mabo P, Victor F, Bazin P, et al. A randomized trial of long-term remote monitoring of pacemaker recipients (The COMPAS trial). Eur Heart J. 2012; 33: 1105-1111.

6 Varma N, Epstein AE, Irimpen A, et al; TRUST Investigators. Efficacy and safety of automatic remote monitoring for implantable cardioverter-defibrillator follow-up. The Lumos-T Safely Reduces Routine Office Device Follow-Up (TRUST) trial. Circulation. 2010; 122: 325-332.
7 Crossley GH, Boyle A, Vitense H, et al. The CONNECT (Clinical Evaluation of Remote Notification to Reduce Time to Clinical Decision) trial. J Am Coll Cardiol. 2011; 57: 1181-1189.

8 Mabo P, Defay P, Sadoul N, et al. Remote follow-up of patients implanted with an ICD: the prospective randomized EVATEL Study. Heart Rhythm. 2011; 9: S226-S227.

9 Saxon LA, Hayes DL, Gilliam FR, et al. Long-term outcome after ICD and CRT implantation and influence of remote device follow-up. The ALTITUDE survival study. Circulation. 2010; 122: 2359-2367.

10 Hindricks G, Taborski M, Glikson M, et al. Implant-based multiparameter telemonitoring of patients with heart failure (IN-TIME): a randomised controlled trial. N Engl J Med. 2014; 384: 583-590.

11 Lunati M, Gasparini M, Santini M, et al. Follow-up of CRT-ICD: implications for the use of remote follow-up systems. Data from the InSync ICD Italian registry. PACE. 2008; 31: 38-46.

12 Slotwiner D, Varma N, Akar JG, et al. HRS Expert Consensus Statement on remote interrogation and monitoring for cardiovascular implantable electronic devices. Heart Rhythm. 2015; 12: 69-100.

13 Humphreys NK, Lowe R, Rance J, Bennett PD. Living with an implantable cardioverter defibrillator: the patients' experience. Heart Lung. 2016; 45: 34-40.

14 Bunz M, Lenski D, Wedegärtner S, et al. Heart-focused anxiety in patients with chronic heart failure before implantation of an implantable cardioverter defibrillator: baseline findings of the Anxiety-CHF Study. Clin Res Cardiol. 2016; 105: 216-224.

15 Thylén I, Moser DK, Strömberg A, et al. Concerns about implantable cardioverter-defibrillator shocks mediate the relationship between actual shocks and psychological distress. Europace. 2016; 18: 828-835.

16 Bedair R, Babu-Narayan SV, Dimopoulos K, et al. Acceptance and psychological impact of implantable defibrillators amongst adults with congenital heart disease. Int J Cardiol. 2015; 181: 218-224.

17 Landolina M, Perego GB, Lunati M, et al. Remote monitoring reduces healthcare use and improves quality of care in heart failure patients with implantable defibrillators: the evolution of management strategies of heart failure patients with implantable defibrillators (EVOLVO) study. Circulation. 2012; 125: 2985-2992.

18 Calò L, Gargaro A, De Ruvo E, et al. Economic impact of remote monitoring on ordinary follow-up of implantable cardioverter defibrillators as compared with conventional in-hospital visits. A single-center prospective and randomized study. J Interv Card Electrophysiol. 2013; 37: 69-78.

19 Buchta $P$, Tajstra $M$, Kurek A, et al. The impact of remote monitoring of implanted cardioverter-defibrillator (ICD) and cardiac resynchronisation therapy device (CRT-D) patients on healthcare costs in the Silesian population: three-year follow-up. Kardiol Pol. 2017; 75: 573-580.

20 Liberska A, Kowalski 0, Mazurek M, et al. Day by day telemetric care of patients treated with cardiac resynchronization therapy - first Polish experience. Kardiol Pol. 2016; 74: 741-748.

21 Varma N, Piccini JP, Snell J, et al. The relationship between level of adherence to automatic wireless remote monitoring and survival in pacemaker and defibrillator patients. J. Am Coll Cardiol. 2015; 65: 2601-2610.

22 Tajstra M, Sokal A, Gadula-Gacek E, et al. Remote Supervision to Decrease Hospitalization Rate (RESULT) study in patients with implanted cardioverter-defibrillator. Europace. 2020; 22: 769-776.

23 Piotrowicz R, Krzesiński P, Balsam P, et al. Cardiology telemedicine solutions opinion of the experts of the Committee of Informatics and Telemedicine of Polish Society of Cardiology, Section of Non-invasive Electrocardiology and Telemedicine of Polish Society of Cardiology and Clinical Sciences Committee of the Polish Academy of Sciences. Kardiol Pol. 2018; 76: 698-707 